\title{
Cell-autonomous generation of the wave pattern within the vertebrate segmentation clock
}

Laurel A. Rohde ${ }^{1,2, \uparrow}$, Arianne Bercowsky-Rama ${ }^{1, \uparrow}$, Jose Negrete Jr. ${ }^{1,3, \uparrow}$, Guillaume Valentin ${ }^{4}$, Sundar Ram Naganathan ${ }^{1,5}$, Ravi A. Desai ${ }^{2,6}$, Petr Strnad ${ }^{1,7}$, Daniele Soroldoni ${ }^{1,2,8}$, Frank Jülicher ${ }^{3}$, and Andrew C. Oates ${ }^{1,2,5, *}$

${ }^{1}$ Institute of Bioengineering, EPFL; Lausanne, $\mathrm{CH}$

${ }^{2}$ Department of Cell and Developmental Biology, UCL; London, UK

${ }^{3}$ Max Planck Institute for the Physics of Complex Systems; Dresden, DE

${ }^{4}$ Center of PhenoGenomics, EPFL; Lausanne, $\mathrm{CH}$

${ }^{5}$ The Francis Crick Institute; London, UK

${ }^{6}$ Current address: Imperial College London; London, UK

${ }^{7}$ Current address: Viventis Microscopy Sárl; Lausanne, $\mathrm{CH}$

${ }^{8}$ Current address: Vienna BioCenter Core Facilities GmbH; Vienna, AU

$\dagger$ Equal contribution

*Corresponding author. Email: andrew.oates@epfl.ch

\begin{abstract}
Sequential segmentation of the body axis is fundamental to vertebrate embryonic patterning. This relies on the segmentation clock, a multi-cellular oscillating genetic-network, which mainifests as tissue-level kinematic waves of gene expression that arrest at the position of each new segment. How this hallmark wave pattern is generated is an open question. We compare cellular-resolution oscillatory patterns in the embryo to those generated cell-autonomously in culture without extrinsic signals. We find striking similarity, albeit with greater variability in the timing of clock arrest in culture. Our simple physical description of a clock controlled by a noisy cell-intrinsic timer captures these dynamics. We propose the segmentation clock integrates an intrinsic, timer-driven oscillatory program, which underlies the waves and arrest, with extrinsic cues regulating the intrinsic timer's duration and precision.
\end{abstract}

One-sentence Summary: Segmentation clock and wavefront activities underlying tissuelevel wave patterns are cell-autonomous properties in the PSM. 
Rythmic and sequential segmentation of the vertebrate body is an iconic embryonic patterning event that involves wave patterns in a population of cellular genetic-oscillators called the segmentation clock (1). Tissue-level waves of gene expression, emerging from the tailbud (TB), travel anteriorly through the pre-somitic mesoderm (PSM) before arresting at the position of each new morphological segment, termed a somite (2-6). Waves are generated by a global phase shift across the PSM as individual cells slow oscillations before arrest $(3,7,8)$. Yet, whether this underlying cellular behaviour is instructed by extrinsic signals or is cellautonomous remains an open question. Here, we describe oscillation and arrest dynamics in individual cells as they progress from TB to somite in the embryo, then test if these dynamics are reproduced cell-autonomously in culture.

To follow cellular dynamics we used the fluorescently-tagged core clock component Her1YFP, a transcriptional repressor, that was used to define the clock's tissue-level wave pattern in zebrafish (6). Previous descriptions of cellular-level oscillations spanned limited intervals and axial locations $(3,8,9)$. To see the full pattern, we back-tracked individual cells over 7.5 $\mathrm{h}\left(15-28^{\text {th }}\right.$ somite stages) in a freely-growing embryo using light-sheet microscopy (Figs. 1A; S1). Cells constituting the $28^{\text {th }}$ somite originated from the TB, where they showed lowintensity, noisy oscillations. As these cells transitioned into posterior-PSM, their oscillations successively grew in intensity and slowed until arresting in the anterior-PSM (Fig. 1B-D). In contrast, cells only reaching the posterior-PSM in this interval spent longer in the TB, and correspondingly delayed their intensity rise and slowing (Fig. 1B,C,E).

To define the wave arrest at cellular-resolution, we backtracked a formed somite (SI) (10). All cells expressed Her1-YFP and most produced their last peak in prospective somite-I (S-I), with a phase profile across the rostral-caudal somite axis reflecting the wave's arrival (Fig. 1F,H). We similarly analyzed the signal-onset of a novel Mesp-ba-mKate2 transgene (Fig. S2). Although Mesp genes are not required to position somite boundaries in zebrafish, their expression is a well-established marker for segment polarity $(11,12)$. We detected cells with a Mesp-ba-mKate2 signal-onset (Fig. S3) only in the rostral half of the forming somite (S0), where it remained in SI (Figs. 1G,I). Taken together, individual cells transitioning from TB to PSM successively slowed oscillations, generating a tissue-level wave pattern until arrest in concert with anterior PSM differention into a polarized somite. 


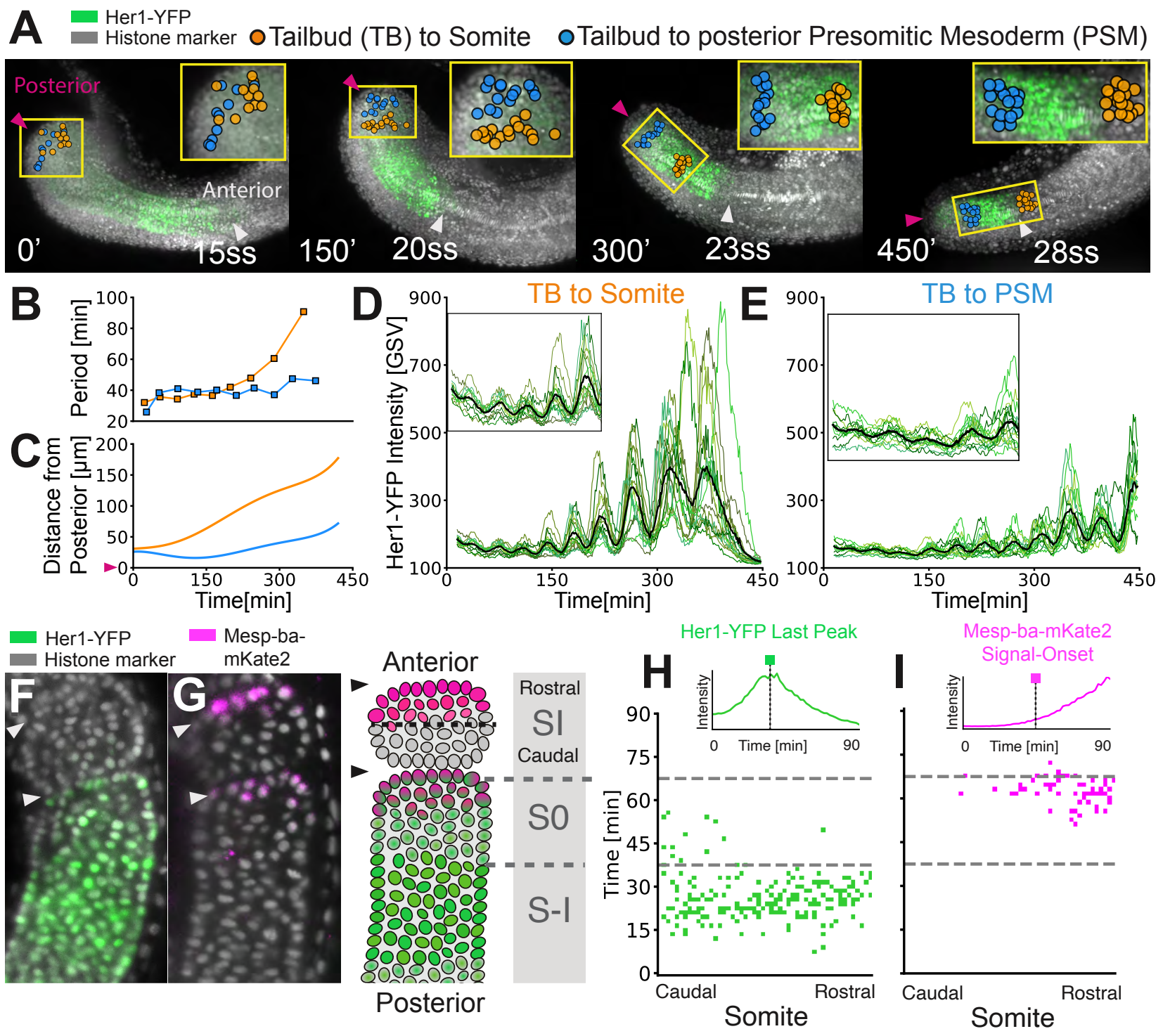

Fig. 1. Transient oscillatory dynamics in the segmentation clock.

(A) Multi-dimensional light-sheet imaging (1.5 min/stack; $7.5 \mathrm{~h})$. Individual cells backtracked from posterior-PSM (blue, $\mathrm{n}=17$ ) and $28^{\text {th }}$ somite (orange, $\mathrm{n}=15$ ) in a $\operatorname{Tg}($ her $1-y f p ; x$ la.eefla1:h2b-mcherry) embryo (SI, white arrowhead; posterior-tip embryo, pink arrowhead). (B) Mean period of Her1-YFP oscillations, and (C) anteroposterior position of cell groups in A. (D, E) Her1-YFP intensity traces for individual cells, mean (black). (F, G) Her1-YFP and Mesp-ba-mKate2 in Tg(her1-yfp;xla.eefla1:h2b-mcherry) and $\operatorname{Tg}$ (mesp-ba-mKate2a;h2a-gfp) embryos (arrowheads at somite boundaries). (H, I) SI backtracked in a $\operatorname{Tg}($ her 1-yfp; $x$ la.eefla1:h2b-mcherry) ( $\mathrm{n}=233$ cells) and Tg(mesp-ba-mKate2a;h2a-gfp) embryo (190 cells). Kymograph of Her1-YFP last peak and Mesp-ba-mKate2 signal-onset in cells relative to rostral-caudal somite axis (example cell trace, inset). 
How are these transient oscillatory dynamics generated? Extrinsic signalling from cell-cell coupling and morphogens can influence PSM length, spatial periodicity of somite formation and rostral-caudal somite polarity in mouse, chick and zebrafish embryos (2, 13-19). Moreover, re-aggregated primary PSM cells, and cultures of induced stem cells eventually generate wave patterns and can segment with rostral-caudal identity, suggesting mammalian PSM cell populations are able to self-organise spatiotemporal information (20-24). Nevertheless, it remains possible that PSM cells carry information from the TB $(13,25-27)$. Consistent with the presence of cell-autonomous information, explanted PSM tissue retains waves and sequentially forms polarized segments $(5,28,29)$. Culture conditions maintaining persistent noisy oscillations have been identified for single PSM cells (20, 21, 30, 31). However, to address whether transient dynamics are generated cell-autonomously or depend solely upon extrinsic instruction from pre-existing or collectively emerging signals requires a single-cell assay permitting PSM differentiation.

To test for cell-autonomous transient dynamics, we followed Her1-YFP and Mesp-ba-mKate2 simultaneously in single primary PSM cells cultured at low-density without added morphogens, serum, BSA, or small molecule inhibitors, thus excluding cell-cell communication and long-range signals (Fig. 2A). We dissected out the posterior-most quarter of the PSM (PSM4) from individual Tg(her1-yfp;mesp-ba-mkate2) embryos, then analyzed single cells remaing alone in the field of view, undivided and alive $5 \mathrm{~h}$ post-dissociation in culture (Figs. 2B,C; S4). Most PSM4 cells showed Her1-YFP oscillations, with 1 to 8 peaks, before arresting (Fig. 2D,E; Table S1). Successive oscillation cycles typically slowed and increased amplitude before arrest (Fig. 2F). Oscillation arrest, as marked by the Her1-YFP last peak, was associated with Mesp-ba-mKate2 signal-onset (Figs. 2C,D; S3), linking it to the position of clock arrest observed in the embryo. This autonomous behaviour was independent of cell survival time in culture (Fig. S5), reproduced in cells isolated one-per-well (Fig. S6) and independent of $T g$ (mesp-ba-mKate2) (Fig. S7). The variability in arrest time was not due to differences between individual embryos and experiments (Fig. S8). Our results indicate that information carried by PSM cells is sufficient to slow oscillations and arrest the clock in concert with switching on a segmental marker.

To compare cell-autonomous dynamics to those in the embryo, we tracked cells starting within PSM4 until somite formation. These cells contributed predominantly to two somites, retaining their intial anterioposterior arrangement, and cells in the same somite differed by at most one 

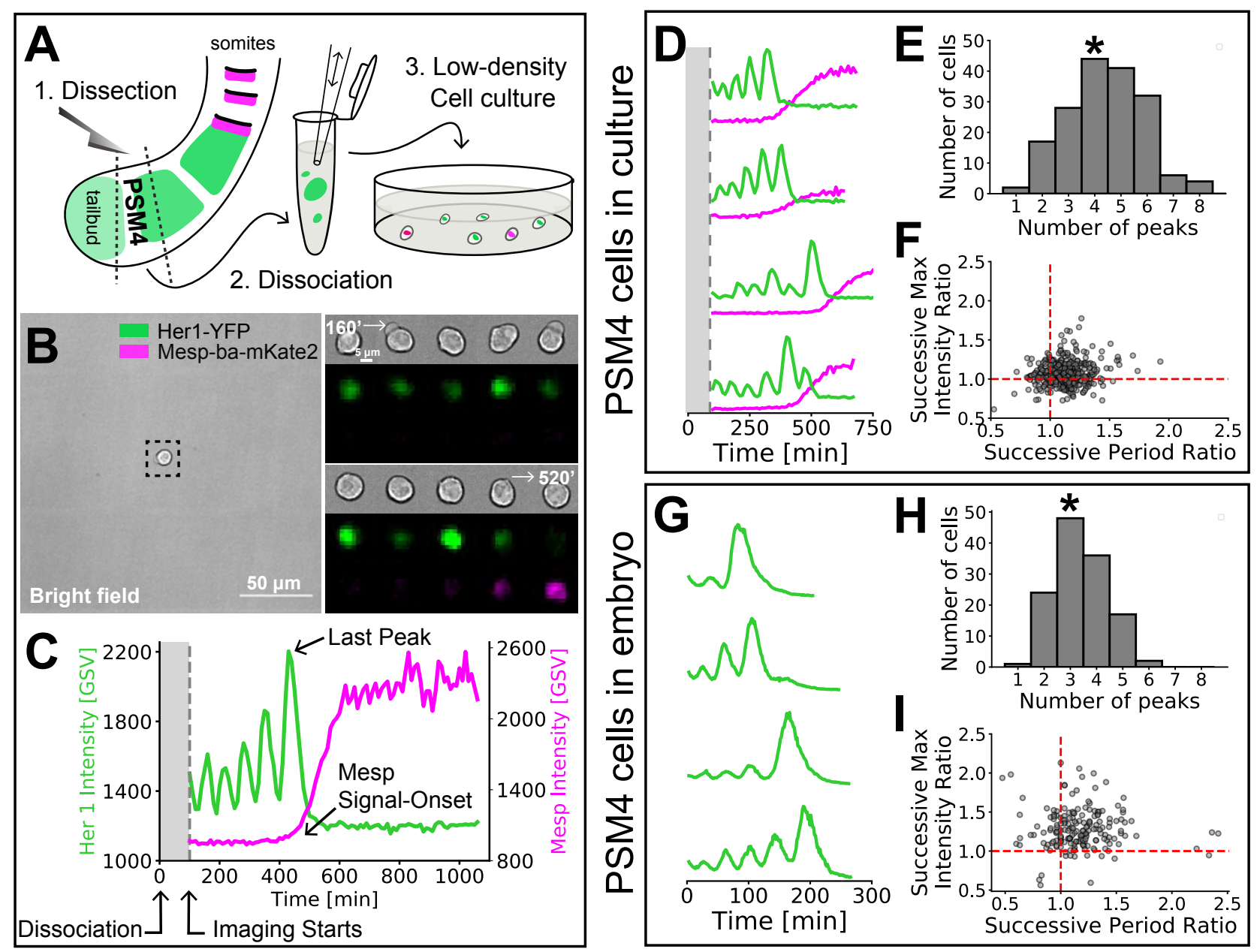

Fig. 2. Cell-autonomous transient oscillatory and differentiation dynamics.

(A) Posterior-PSM quarter (PSM4) dissected from a Tg(her1-yfp;mesp-ba-mKate2) 15 somite-stage embryo, dissociated into single cells, then cultured at low density. (B) Time-lapse of single cell. (C) Intensity trace of cell in B. (D) Representative Her1-YFP/Mesp-ba-mKate2 intensity traces from PSM4 cells ( $\mathrm{N}=11$ embryos, $\mathrm{n}=174$ cells). (E, H) Her1-YFP peaks percell (*mean $\pm \mathrm{SD}, 4.4 \pm 1.6$ peaks in culture, $3.4 \pm 1.0$ peaks in embryo). (F, I) Successive peak (maxima) intensity and period ratios ( $\mathrm{n}=421$ cycles, 55\% cycles in upper-right quadrant (F); $\mathrm{n}=179$ cycles, $72 \%$ cycles in upper-right quadrant (I)). (G) Her1-YFP intensity traces from PSM4 cells tracked in $T g$ (her $1-y f p ;$;2b-mcherry) embryos ( $\mathrm{N}=2$ embryos, $\mathrm{n}=133$ PSM4 cells). 
peak (Fig. S9). As in culture, we only analyzed undivided cells (Figs. 2G;S10), and normalized for a general slowing of developmental time observed in zebrafish culture (Fig. S11) $(31,32)$ by comparing the ratio of successive periods and peak intensities, and number of peaks rather than absolute time (Fig. 2H,I). PSM4 cells in culture generated more peaks, with more variability than we observed in the embryo (Fig 2 E,H), although both successivly slowed and increased intensity (Fig. 2F,I). We detected Mesp-ba-mKate2 signal-onset in the same percentage of PSM4 cells in culture as the embryo's formed somite (Figs. 1G,I;2D; S3). However, the cell-autonomous Mesp-ba-mKate2 signal-onset spanned the last peak rather than occurring only afterwards (Figs. 1H,I; S3). Taken together, longer-running and noisier dynamics in culture suggest that extrinsic information in the PSM acts to shorten and sharpen a cell-instrinsic program.

Our discovery of cell-autonomous transient dynamics leads to the hypothesis that cells encode information about their position along the PSM using an intrinsic timing mechanism. This mechanism is likely independent of the clock, as we found comparable cell-autnomous timing of Mesp-ba-mkate2 signal-onset with wildype and disabled clock oscillations (her $1^{-/-} ;$her $^{7^{-/}}$) (S12) (33). An intrinsic timer hypothesis predicts that PSM cells at different anteroposterior locations will have run down their timer to different extents, such that more anterior cells will arrest oscillations and differentiate earlier in culture. To test this, we followed autonomous dynamics in cells from the TB and different PSM quarters (Fig. 3A). Consistent with an intrinsic timer, we found that PSM cells originating more anteriorly tended to arrest Her1-YFP oscillations earlier, in concert with earlier Mesp-ba-mKate2 signal-onset (Figs. 3A-C; S13AF).

In contrast to cells remaining within the TB of the embryo, which maintained low-intensisty, noisy oscillations (Figs. 1A; S1), TB cells removed into culture successively slowed oscillations and increased peak intensity (Fig. S13C), until arresting together with Mesp-bamKate2 signal-onset at a similar time to PSM4 cells taken from the same embryos (Figs. 3B,C; $\mathrm{S} 13 \mathrm{~A}, \mathrm{~F})$. This differs from the persistent noisy oscillations seen in previous cultures of single TB cells in serum and FGF (31). We propose that TB cells carry the timing mechanism, but a trigger associated with exit from the TB, either transition to PSM in the embryo or removal into cell culture, is required to start the timer. The role of extrinsic factors in the TB, such as FGF and Wnt (34), may thus be to prevent the timer from initiating. 

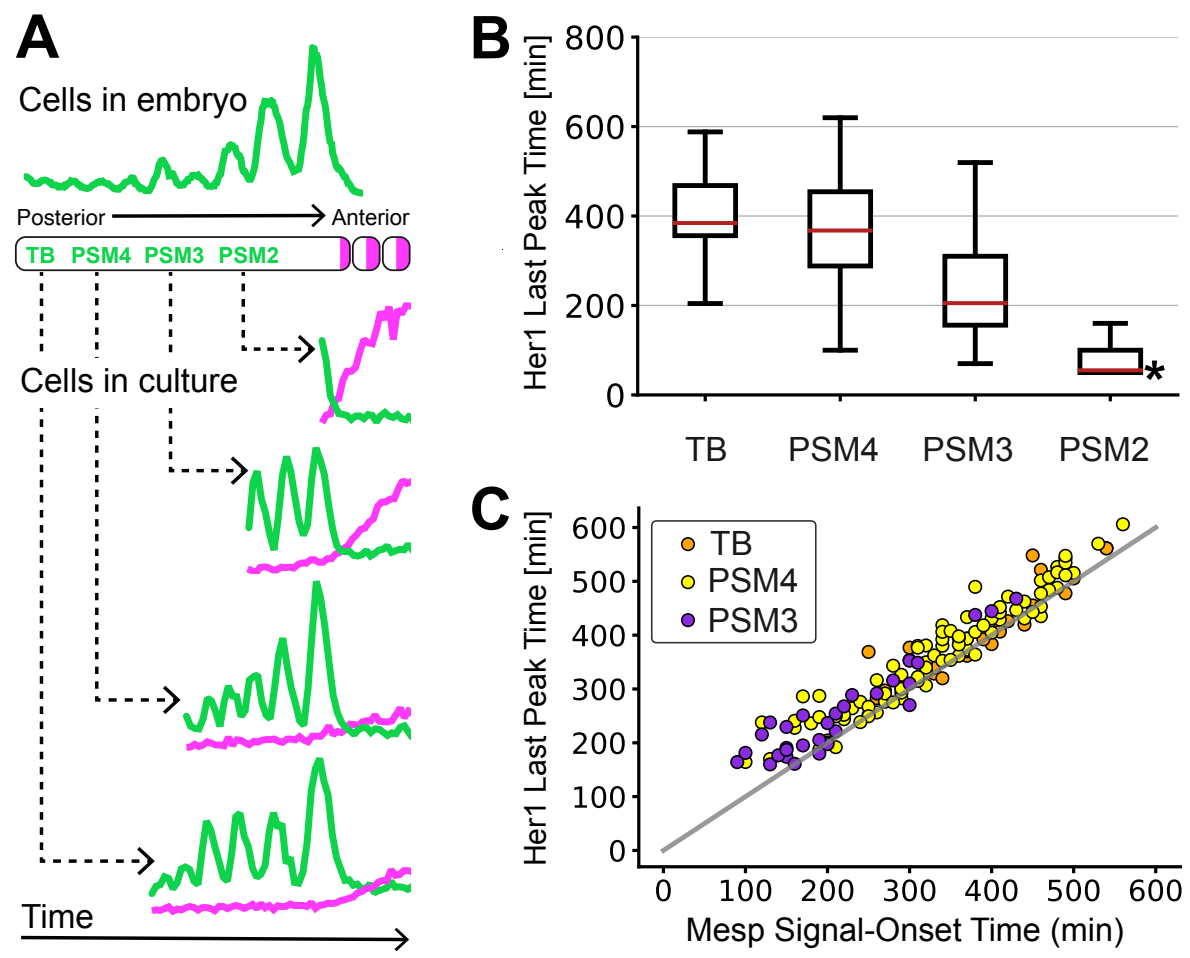

Fig. 3. Cell-intrinsic timing runs down across PSM.

(A) Experiment schematic of cultured cells from TB and three PSM quarters (Tg(her1-yfp;me$s p$-bb-mKate2). Representative intensity traces versus the full anteroposterior pattern in embryo (Fig. 1D), aligned by last peak. (B) Her1-YFP last peak time post-dissociation in culture (median, interquartile box, whiskers). PSM4 from each embryo was cultured in parallel as an internal reference then data pooled (9 embryos; 32 PSM2, 65 PSM3 and 132 PSM4 cells). If PSM2 had a last peak pre-acquisition (example in A), its time was set to the imaging start (*). (C) Correlation of Her1-YFP last peak and Mesp-bb-mKate2 signal-onset time, grey line shows identity. 
If such a cell-instrinsic timer were directly regulating oscillation and arrest dynamics, we reasoned that it should leave a signature in the Her1-YFP oscillatory profile in culture. We aligned Her1-YFP intensity traces from cultured PSM4 cells by the first observable cycle, then analyzed peak (I+) and trough (I-) intensities, and production (T+) and degradation ( $\mathrm{T}-$ ) time intervals throughout successive cycles (Fig. 4A). I+ increased and I- fluctuated around a constant value (Fig. 4B), the period consistenly lengthed, and an asymmetry was apparent between T+ and T- (Fig. 4C). Importantly, this asymmetric signature implies that oscillations are slowed across the PSM by increasing Her1 production rate over time (Supplementary Text 1 and 2), and suggests the intrinsic timer acts by regulating the clock's production rate.

Considering this signature, we captured the functional form of an intrinsic timer in a simple model for externally regulated genetic oscillations (35). The genetic clock $I(t)$, which represents Her1 dynamics, is regulated by the timer through the clock's time-dependent production rate $P(t)$ (Fig. 4D). The clock is described by a stochastic differential equation with time-delayed negative feedback, subject to multiplicative noise. In the timer, a time-keeping activity increases linearly until a threshold, then undergoes rapid inactivation (Fig. 4E). Accordingly, intensity of the oscillatory profile increases monotonically, then arrests back to equilibrium. To mimic biochemical noise in the timer, Brownian noise generates a noisy ramp with intrinsic variability in the time to reach the inactivation threshold (Fig. 4E), and corresponding variability in the duration of resulting transient oscillatory dynamics (Fig. 4F).

This model predicts that I- has a narrow probability distribution, fluctuating around a constant value as timer activity increases (Fig. 4G). In contrast, I+ has a direct dependence on timer activity, resulting in a broader probability distribution. Also predicted is an asymmetry in the statistics of $\mathrm{T} \pm$ (Fig. $4 \mathrm{H})$, where $\mathrm{T}$ - correlates with initial $\mathrm{I}+$, since longer decay times correspond to larger peaks, while $\mathrm{T}+$ is relatively insensitive to initial I- (35). Using the distribution of I \pm obtained from PSM4 cells in culture, we fit the model (Fig. 4G). The resulting probability distributions for $\mathrm{T} \pm$ are a direct consequence of this fit (Fig. 4H). Agreement between experiment and simulation indicates that a noisy ramp in the clock's production rate $P(t)$ faithfully captures the statistics of the cell-autonomous transient dynamics. Agreement was not found by modulating the binding constants $\left(K_{0}\right)$, the delay $\left(t_{d}\right)$ or degradation $\left(T_{0}\right)$ times in the clock (Supplementary Text 1 and 2, Fig. S14). This supports the hypothesis of a 
A Oscillatory Profile
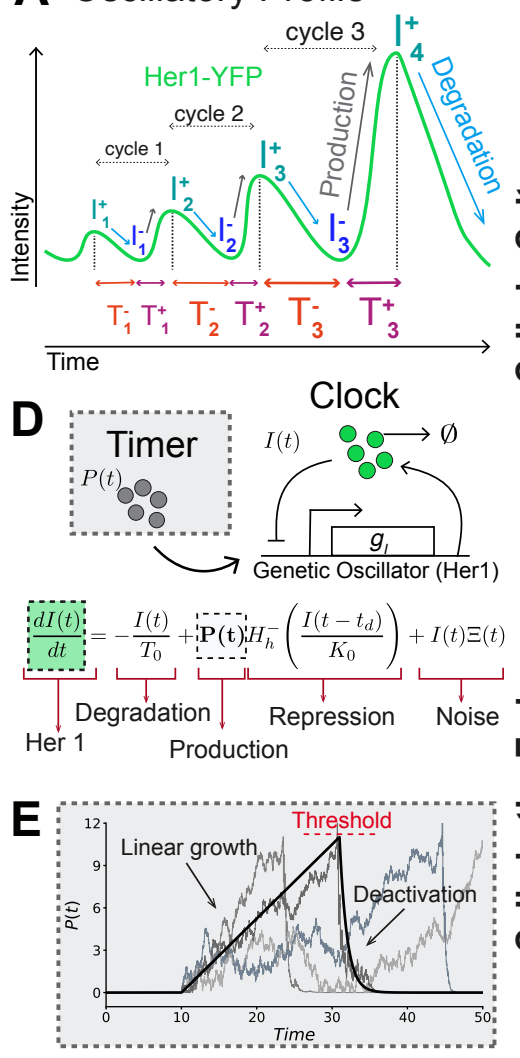

Experiment

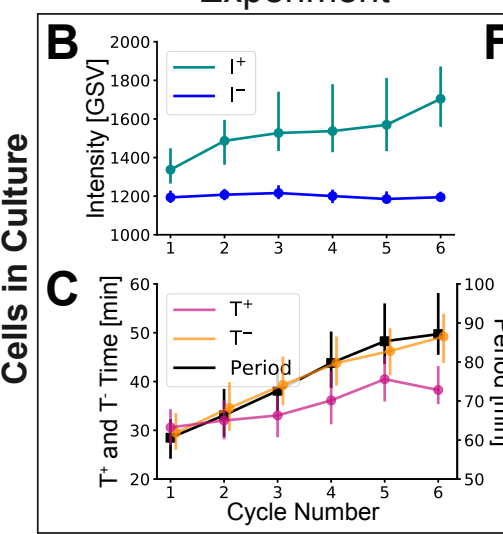

Simulation

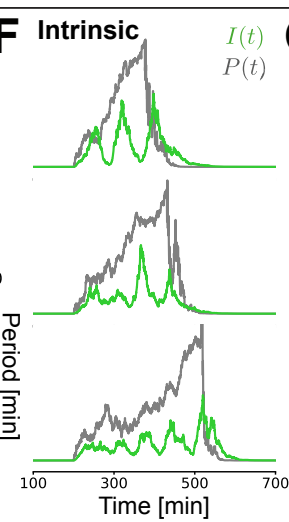

$I(t)$
$P(t)$
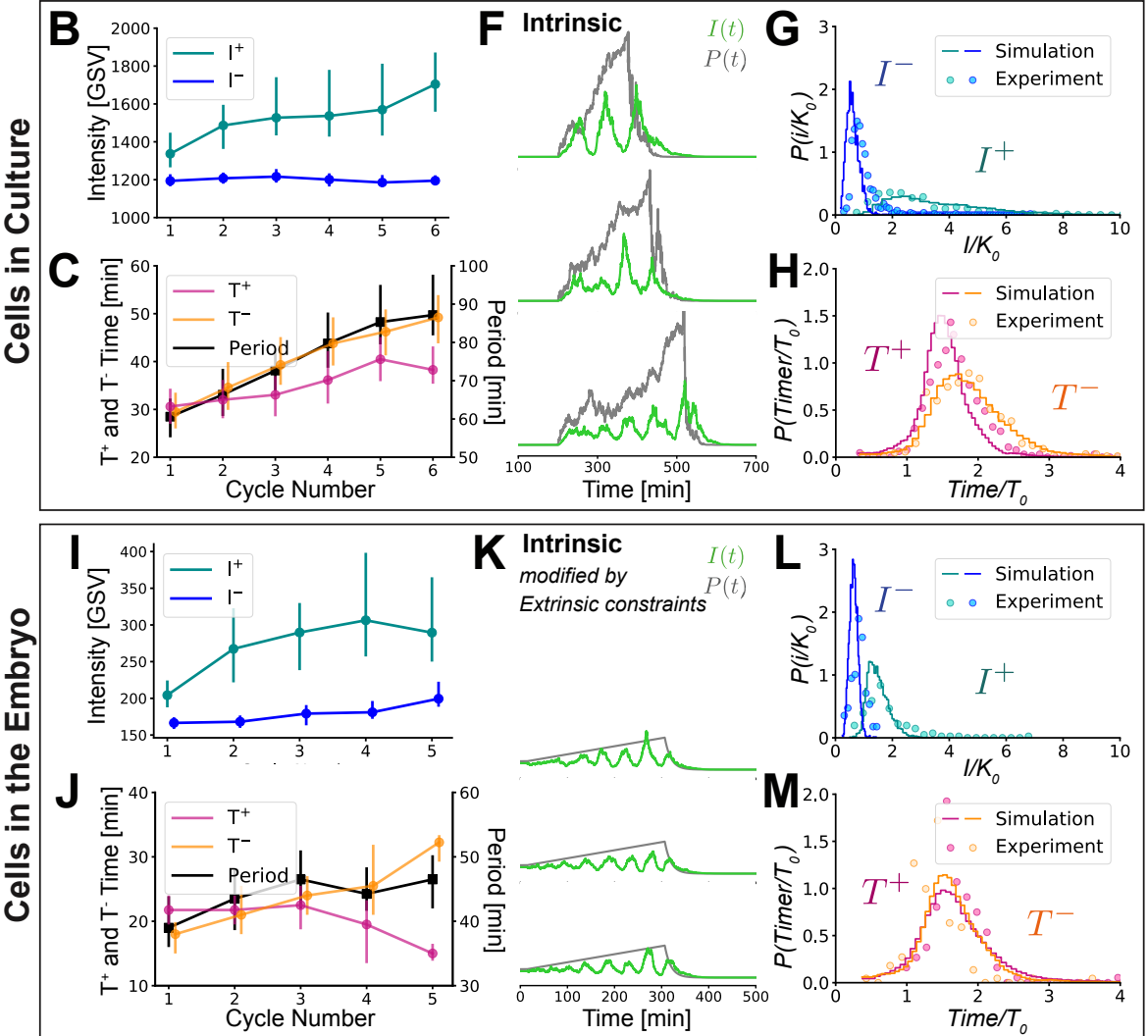

Fig. 4. Oscillatory profile reveals signature of cell-intrinsic timing mechanism

(A) Observables of Her1-YFP profile: peak (I+) and trough (I-) intensity; time-intervals of production $(\mathrm{T}+)$ and degradation (T-). (B, C) Cell-autonomous PSM4 observables aligned by first cycle. (D) Schematic and clock equation of model. (E) Production rate $P(t)$ acts as a noisy timer. (F) Oscillatory (green) and noisy ramp (grey) representative traces from numerical simulations of intrinsic program. (G, H) Probability distributions of observables for cultured PSM4 cells (circles) and intrinsic program simulations (lines). (I, J) Observables from PSM4 cells in embryo aligned by first cycle. (K) As in F, but modified by intrinsic constraints. (L, M) As in $\mathrm{G}, \mathrm{H}$, using cells in the embryo. 
noisy cell-intrinsic timer, and indicates that modulation of the segmentation clock's production rate by the timer is a mechanism consistent with the experimental data.

We noticed that the profile of $\mathrm{I} \pm$ and $\mathrm{T} \pm$ differed between PSM4 cells in culture and in the embryo (Fig. 4B,C,I,J). Using our model we sought to learn how extrinsic PSM signals could influence the cell-intrinsic timer. We found that the oscillatory profile and transient dynamics statistics observed in the embryo were captured in the model by changing timer parameters (Fig. 4K-M) to alter the clock's production rate, without changing other clock parameters (Supplementary Text 2, Fig. S14). We infer from this fit that extrinsic signals in the PSM reduce the timer's production rate and threshold, in addition to strongly reducing its noise.

Together, our experimental observations and theoretical modelling indicate that cells transitioning from TB into PSM start a noisy cell-intrinsic timer, distinct from the clock circuit. This timer runs down during the anterior-ward cell flow, thereby creating spatial information across the PSM. Extrinsic information, through mechanisms such as diffusible morphogen signalling gradients or intercellular communication, is not required for the intrinsic dynamics. Rather, the role of extrinsic factors in the PSM appears restricted to fine-tuning the duration and precision of the intrinsic program. The running down of an intrinisic timer along the PSM fits with previous models of signal gradients established by inheritance of a factor in the posterior that decays as a cell flows $(13,25-27)$, but the intrinsic timer's identity and how it is tuned remain intriguing questions. The rise-and-fall profile of the transient dynamics rules out the simplest scenario of decay of a single time-keeping factor that was inherited by cells in the TB $(25,26)$, as a decay mechanism could directly generate only the rise (or fall) part of the profile, but not both. Instead, the observed dynamics suggest a PSM-specific system that actively initiates as the cells leave the TB.

Our model for the timer is phenomenological, describing only the functional output onto the clock's oscillations. The internal details of such a system are not constrained by our data, and could involve a number of plausible intermediates such as transcription factor or microRNA cascades similar to those in timing of neuroblast differentiation or C. elegans molting $(36,37)$ or phospho-timers as found in the circadian clock (38). The latter mechanism would provide an attractive link to extrinsic signalling by FGF gradients in the PSM $(18,19)$. The timer's 
proposed role in slowing and then stopping oscillations via a rise and fall of Her1 production suggests that factors controlling herl transcription and/or Her1 translation are involved.

In summary, our findings in zebrafish suggest that a picture of the segmentation clock as a cellular population composed of persistent oscillators that are instructed by extrinsic signals is inadequate. We point instead to a central role played by cell-intrinsic programs of timed transient dynamics and differentiation that are extrinsically modulated during embryonic patterning (39). The long-standing and influential "clock and wavefront" model posited an independent wavefront in the tissue, able to cause a sudden transition where locally coherent oscillations were used to determine which cells would segment together (40). Our work shows that both clock and wavefront activities are autonomous properties of PSM cells. This opens the door to a range of experiments using this new assay system, in which the cell intrinsic program can be investigated, as well as challenged with extrinsic signals along its time-course to better understand the control of wave patterns in the segmentation clock. 
1. A. C. Oates, L. G. Morelli, S. Ares, Patterning embryos with oscillations: structure, function and dynamics of the vertebrate segmentation clock. Development. 139, 625-639 (2012).

2. A. Aulehla, W. Wiegraebe, V. Baubet, M. B. Wahl, C. Deng, M. Taketo, M. Lewandoski, O. Pourquié, A $\beta$-catenin gradient links the clock and wavefront systems in mouse embryo segmentation. Nature Cell Biology. 10, 186-193 (2008).

3. E. A. Delaune, P. François, N. P. Shih, S. L. Amacher, Single-Cell-Resolution Imaging of the Impact of Notch Signaling and Mitosis on Segmentation Clock Dynamics. Developmental Cell. 23, 995-1005 (2012).

4. Y. Masamizu, T. Ohtsuka, Y. Takashima, H. Nagahara, Y. Takenaka, K. Yoshikawa, H. Okamura, R. Kageyama, Real-time imaging of the somite segmentation clock: revelation of unstable oscillators in the individual presomitic mesoderm cells. Proceedings of the National Academy of Sciences. 103, 1313-1318 (2006).

5. I. Palmeirim, D. Henrique, D. Ish-Horowicz, O. Pourquié, Avian hairy gene expression identifies a molecular clock linked to vertebrate segmentation and somitogenesis. Cell. 91, 639-648 (1997).

6. D. Soroldoni, D. J. Jorg, L. G. Morelli, D. L. Richmond, J. Schindelin, F. Julicher, A. C. Oates, A Doppler effect in embryonic pattern formation. Science. 345, 222-225 (2014).

7. L. G. Morelli, S. Ares, L. Herrgen, C. Schröter, F. Jülicher, A. C. Oates, Delayed coupling theory of vertebrate segmentation. HFSP Journal. 3, 55-66 (2009).

8. N. P. Shih, P. Francois, E. A. Delaune, S. L. Amacher, Dynamics of the slowing segmentation clock reveal alternating two-segment periodicity. Development. 142, 1785-1793 (2015).

9. K. Yoshioka-Kobayashi, M. Matsumiya, Y. Niino, A. Isomura, H. Kori, A. Miyawaki, R. Kageyama, Coupling delay controls synchronized oscillation in the segmentation clock. Nature. 580, 119-123 (2020).

10. O. Pourquié, P. P. L. Tam, A Nomenclature for Prospective Somites and Phases of Cyclic Gene Expression in the Presomitic Mesoderm. Developmental Cell. 1, 619-620 (2001).

11. S. J. Cutty, R. Fior, P. M. Henriques, L. Saúde, F. C. Wardle, Identification and expression analysis of two novel members of the Mesp family in zebrafish. Int. J. Dev. Biol. 56, 285-294 (2012).

12. T. Yabe, K. Hoshijima, T. Yamamoto, S. Takada, Quadruple zebrafish mutant reveals different roles of Mesp genes in somite segmentation between mouse and zebrafish. Development. 143, 2842-2852 (2016).

13. L. Bajard, L. G. Morelli, S. Ares, J. Pecreaux, F. Julicher, A. C. Oates, Wnt-regulated dynamics of positional information in zebrafish somitogenesis. Development. 141, 1381-1391 (2014).

14. J. Dubrulle, M. J. McGrew, O. Pourquié, FGF Signaling Controls Somite Boundary Position and Regulates Segmentation Clock Control of Spatiotemporal Hox Gene Activation. Cell. 106, 219$232(2001)$.

15. L. Herrgen, S. Ares, L. G. Morelli, C. Schröter, F. Jülicher, A. C. Oates, Intercellular Coupling Regulates the Period of the Segmentation Clock. Current Biology. 20, 1244-1253 (2010). 
16. I. H. Riedel-Kruse, C. Muller, A. C. Oates, Synchrony Dynamics During Initiation, Failure, and Rescue of the Segmentation Clock. Science. 317, 1911-1915 (2007).

17. D. W. K. Sari, R. Akiyama, H. Naoki, H. Ishijima, Y. Bessho, T. Matsui, Time-lapse observation of stepwise regression of Erk activity in zebrafish presomitic mesoderm. Scientific Reports. 8 (2018), doi:10.1038/s41598-018-22619-9.

18. A. Sawada, M. Shinya, Y.-J. Jiang, A. Kawakami, A. Kuroiwa, H. Takeda, Fgf/MAPK signalling is a crucial positional cue in somite boundary formation. Development. 128, 4873-4880 (2001).

19. M. F. Simsek, E. M. Özbudak, Spatial Fold Change of FGF Signaling Encodes Positional Information for Segmental Determination in Zebrafish. Cell Reports. 24, 66-78.e8 (2018).

20. M. Diaz-Cuadros, D. E. Wagner, C. Budjan, A. Hubaud, O. A. Tarazona, S. Donelly, A. Michaut, Z. Al Tanoury, K. Yoshioka-Kobayashi, Y. Niino, R. Kageyama, A. Miyawaki, J. Touboul, O. Pourquié, In vitro characterization of the human segmentation clock. Nature. 580, 113-118 (2020).

21. A. Hubaud, I. Regev, L. Mahadevan, O. Pourquié, Excitable Dynamics and Yap-Dependent Mechanical Cues Drive the Segmentation Clock. Cell (2017), doi:10.1016/j.cell.2017.08.043.

22. M. Matsuda, Y. Yamanaka, M. Uemura, M. Osawa, M. K. Saito, A. Nagahashi, M. Nishio, L. Guo, S. Ikegawa, S. Sakurai, S. Kihara, T. L. Maurissen, M. Nakamura, T. Matsumoto, H. Yoshitomi, M. Ikeya, N. Kawakami, T. Yamamoto, K. Woltjen, M. Ebisuya, J. Toguchida, C. Alev, Recapitulating the human segmentation clock with pluripotent stem cells. Nature. 580, 124-129 (2020).

23. M. Matsumiya, T. Tomita, K. Yoshioka-Kobayashi, A. Isomura, R. Kageyama, ES cell-derived presomitic mesoderm-like tissues for analysis of synchronized oscillations in the segmentation clock. Development. 145, dev156836 (2018).

24. C. D. Tsiairis, A. Aulehla, Self-Organization of Embryonic Genetic Oscillators into Spatiotemporal Wave Patterns. Cell. 164, 656-667 (2016).

25. A. Aulehla, C. Wehrle, B. Brand-Saberi, R. Kemler, A. Gossler, B. Kanzler, B. G. Herrmann, Wnt3a plays a major role in the segmentation clock controlling somitogenesis. Developmental cell. 4, 395-406 (2003).

26. J. Dubrulle, O. Pourquié, fgf 8 mRNA decay establishes a gradient that couples axial elongation to patterning in the vertebrate embryo. Nature. 427, 419-422 (2004).

27. S. J. Gaunt, D. Drage, A. Cockley, Vertebrate caudal gene expression gradients investigated by use of chick cdx-A/lacZ and mouse cdx-1/lacZ reporters in transgenic mouse embryos: evidence for an intron enhancer. Mechanisms of Development. 120, 573-586 (2003).

28. M. Maroto, J. K. Dale, M.-L. Dequeant, A.-C. Petit, O. Pourquie, Synchronised cycling gene oscillations in presomitic mesoderm cells require cell-cell contact. Int. J. Dev. Biol. 49, 309-315 (2005).

29. I. Palmeirim, J. Dubrulle, D. Henrique, D. Ish-Horowicz, O. Pourquié, Uncoupling segmentation and somitogenesis in the chick presomitic mesoderm. Developmental Genetics. 23, 77-85 (1998). 
30. M. Matsuda, H. Hayashi, J. Garcia-Ojalvo, K. Yoshioka-Kobayashi, R. Kageyama, Y. Yamanaka, M. Ikeya, J. Toguchida, C. Alev, M. Ebisuya, Species-specific segmentation clock periods are due to differential biochemical reaction speeds. Science. 369, 1450-1455 (2020).

31. A. B. Webb, I. M. Lengyel, D. J. Jörg, G. Valentin, F. Jülicher, L. G. Morelli, A. C. Oates, Persistence, period and precision of autonomous cellular oscillators from the zebrafish segmentation clock. eLife. 5, e08438 (2016).

32. T. Langenberg, M. Brand, M. S. Cooper, Imaging brain development and organogenesis in zebrafish using immobilized embryonic explants. Dev. Dyn. 228, 464-474 (2003).

33. L. Lleras Forero, R. Narayanan, L. F. Huitema, M. VanBergen, A. Apschner, J. Peterson-Maduro, I. Logister, G. Valentin, L. G. Morelli, A. C. Oates, S. Schulte-Merker, Segmentation of the zebrafish axial skeleton relies on notochord sheath cells and not on the segmentation clock. eLife. 7, e33843 (2018).

34. B. L. Martin, Factors that coordinate mesoderm specification from neuromesodermal progenitors with segmentation during vertebrate axial extension. Seminars in Cell \& Developmental Biology. 49, 59-67 (2016).

35. J. Negrete JR, I. M. Lengyel, L. A. Rohde, R. A. Desai, A. Oates, F. Jülicher, Theory of time delayed genetic oscillations with external noisy regulation. New J. Phys. (2021), doi:10.1088/1367-2630/abd80b.

36. V. Ambros, MicroRNAs and developmental timing. Current Opinion in Genetics \& Development. 21, 511-517 (2011).

37. T. Brody, W. F. Odenwald, Programmed Transformations in Neuroblast Gene Expression during Drosophila CNS Lineage Development. Developmental Biology. 226, 34-44 (2000).

38. A. C. R. Diernfellner, M. Brunner, Phosphorylation Timers in the Neurospora crassa Circadian Clock. Journal of Molecular Biology. 432, 3449-3465 (2020).

39. M. H. Syed, B. Mark, C. Q. Doe, Playing Well with Others: Extrinsic Cues Regulate Neural Progenitor Temporal Identity to Generate Neuronal Diversity. Trends in Genetics. 33, 933-942 (2017).

40. J. Cooke, E. C. Zeeman, A clock and wavefront model for control of the number of repeated structures during animal morphogenesis. Journal of theoretical biology. 58, 455-76 (1976).

Acknowledgements: We thank the following: A. Aulehla, M. Ebisuya, J. Garcia Ojalvo, A. Martinez Arias, G. Mönke, L. Morelli, C. Mulas, B. Steventon, K. Uriu, and Oates lab members for comments on the manuscript; J-Y Tinevez and T. Pietzsch for Mastodon assistance; MPI-CBG, UCL, and EPFL fish facilities; D. Rohde for an X; U. Schulze and A. Boni for imaging help; and C. Jollivet and V. Sergy for support.

Funding: We received funding from EPFL the Francis Crick Institute and Max-Planck-Gesellshaft. SNSF Project funding division III (31003A_176037) to JN and AO. Wellcome Trust Senior Research Fellowship in Basic Biomedical Science (WT098025MA) to AO. MPIPKS Visitors Program to JN. 
Long-Term Human Frontier Science Program postdoctoral fellowship (LT000078/2016) to SRN. Whitaker International Fellowship to RAD.

\section{Author contributions:}

Conceptualization: LAR, JN, ABR, ACO

Methodology: LAR, ABR, JN, GV, SRN, RAD, PS, DS

Investigation: LAR, ABR, JN, GV, SRN, RAD

Visualization: ABR, LAR, JN, ACO

Software: ABR, SRN, JN, PS

Funding acquisition: FJ, ACO

Supervision: FJ, ACO

Writing - Original Draft Preparation: LAR

Writing - Review \& Editing: LAR, ABR, JN, ACO

Competing Interests: Authors declare that they have no competing interests.

Data and materials availability: All data, code, and materials available upon request.

\section{Supplementary Materials:}

Materials and Methods

Supplementary Text 1

Supplementary Text 2

Figures S1 to S14

Table S1 